\title{
LA EXTENSIÓN UNIVERSITARIA Y SU GESTIÓN PEDAGOGICA EN LA UNIVERSIDAD CUBANA
}

\section{UNIVERSITY EXTENSION AND ITS PEDAGOGICAL MANAGEMENT IN CUBAN UNIVERSITY}

\author{
Dagneris Batista de Los Ríos* \\ Yanet Trujillo Balboquin** \\ Yoenia Virgen Barbán Sarduy***
}

Resumen: En la investigación se ofrece una sistematización teórica del proceso de extensión universitaria y su gestión a partir de la dimensión pedagógica, se definen sus categorías fundamentales y la necesaria interrelación con los procesos académico, investigativo y laboral. La nueva concepción de la gestión pedagógica de este proceso en las universidades, favorece una mejor preparación de los profesores, la transformación de los sujetos, los contextos, y la formación integral del estudiante que le permitirá dar respuestas a las exigencias sociales.

Palabras clave: Extensión universitaria; gestión; formación integral.

\begin{abstract}
This study presents the theoretical systematization of university extension and its management process taking into account pedagogical dimension. It defines its fundamental categories and the necessary interrelationship between academic, investigative and labor demands. The use of pedagogical management in universities improves teacher education, transforms the subjects and contexts, and provides integral education to students, preparing them to deal with social demands.
\end{abstract}

Keywords: University extension; management; integral training. 


\section{Introducción}

El avance acelerado del desarrollo científicotecnológico en la actualidad genera transformaciones en todas las esferas de la vida social, especialmente en la educación. Las universidades necesitan actualizar sus programas de estudio y procesos académicos, renovar sus prácticas, para lograr la formación de profesionales capaces de enfrentar los cambios y dar respuesta a las exigencias sociales.

En el artículo 39 de la Constitución de la República de Cuba, en su capítulo V sobre Educación y Cultura se estipula: "el Estado orienta, fomenta y promueve la educación, la cultura y las ciencias en todas sus manifestaciones" (1992:19). Se fundamenta en los avances de la ciencia y la técnica, el ideario marxista y martiano, la tradición pedagógica progresista cubana y universal, la combinación de la educación con el trabajo, la investigación para el desarrollo, el deporte, la participación en actividades políticas, sociales, aspectos esenciales de la extensión universitaria.

Las universidades asumen una nueva significación estratégica en la formación de recursos humanos, generación de conocimientos, el intercambio cultural con la sociedad, perfeccionamiento de sus procesos académicos, investigación y extensión universitaria, aspectos de relevancia en esta tesis. En la extensión universitaria el proceso de gestión permite evaluar la pertinencia, impacto y relevancia de la universidad en la sociedad, su objetivo fundamental es desarrollar la cultura universitaria, que se constituye a partir de la cultura académica, científica, económica, política, medioambiental, de salud, preventiva, patrimonial, artística, deportiva, de la profesión, entre otros aspectos esenciales en la formación inicial.

El proceso de extensión universitaria desempeña un papel primordial en la solución a las exigencias sociales, al tener en cuenta las peculiaridades de cada carrera, en correspondencia con la formación profesional del estudiante universitario. Se desarrolla al estudiante ingresar en la universidad, pero antes se realiza mediante actividades de formación vocacional en enseñanzas precedentes, para motivarlos a matricular en carreras necesarias para la sociedad.

El estudio de las limitaciones en la práctica y en la teoría permitió determinar que persisten concepciones que circunscriben la gestión de extensión universitaria a la dimensión administrativa, persiste el desconocimiento de elementos pedagógicos que tributan a elevar la gestión y en la formación integral del estudiante.

\section{Desarrollo}

1.1 - Fundamentos pedagógicos del proceso de extensión universitaria

En la Primera Conferencia Latinoamericana de Extensión Universitaria y Difusión Cultural en 1957, se expresó que por su naturaleza, es misión y función orientadora de la universidad contemporánea, ejercicio de la vocación universitaria. Por su contenido y procedimientos se funda en estudios y actividades filosóficas, científicas, artísticas y técnicas. Por su finalidad debe proyectar dinámica y coordinadamente la cultura, vincular al pueblo con la universidad para elevar su nivel espiritual, intelectual y técnico. Este proceso estimula el desarrollo al dinamizar la relación entre universidad y sociedad.

González, M., González, G. R. (2013), sustentan que en la extensión universitaria se da una relación dialéctica función-proceso con carácter de ley, es un sistema de interacciones entre la universidad y la sociedad mediante la actividad y la comunicación, parte de una planificación estratégica y unidades de acción desde la formación curricular, la superación, difusión de los resultados de la ciencia, proyectos de investigación, socioculturales, actividades extracurriculares, con el propósito de promover la cultura. Sin embargo, aún no se logra articular los procesos académico, investigativo y laboral, lo que incide en el cumplimiento de los objetivos educativos y la formación integral del estudiante.

Tunnerman, C. (2003) enfatiza que la función de extensión, a la par de la docencia y la investigación, forma parte integral de la misión educativa de las instituciones de educación superior. Deben estar presentes en las políticas y estrategias de las instituciones, interrelacionándose y enriqueciéndose recíprocamente. La concatenación universal está presente en la articulación entre los procesos, se favorecen las funciones instructivas, educativas y de desarrollo, expresión de la relación teoría y práctica, al considerar la teoría como propiedad inherente a la actividad y la práctica como condición de la teoría.

La extensión es asociada a nuevas formas de expresión que incorpora la innovación, la inclusión social, la internacionalización, pasantías estudiantiles, las prácticas pre profesionales, las cuales implican un nuevo rol de los estudiantes en el proceso educativo, y son elementos esenciales en la gestión de la extensión universitaria en los diferentes niveles organizativos, fundamentalmente desde el colectivo de año académico que es el responsable de conducir este proceso.

Del Valle Blohm, N. C. (2009:19) define extensión universitaria como: "un proceso dinámico de formación integradora y sistémica, basada en la interacción cultural del quehacer universitario en comunicación multidireccional permanente con la sociedad, orientando a la transformación social para 
buscar en las comunidades donde desarrolla su acción la solución a sus problemas, responde a necesidades concretas en un momento determinado. Posee objetivos y contenidos propios, se realiza a través de diferentes métodos, necesita de medios, recursos adecuados y mecanismos de planificación y evaluación sistemática para su perfeccionamiento".

Este proceso articula lo instructivo, educativo y desarrollador con otros procesos que se presuponen y excluyen mutuamente; desde la dialéctica materialista son reflejos de la objetividad que interactúan para que ocurra el desarrollo.

En el Congreso Universidad 2014 (2014:4) se plantea: "La extensión universitaria debe concebirse en cuatro dimensiones: la institucional (académica sustantiva), la dialógica, la transformación social y la pedagógica (educación experiencial), se requiere trabajar la extensión universitaria como parte del perfeccionamiento del proceso". Idea que apunta hacia una nueva visión de su gestión, no como proceso administrativo e institucional, sino formativo, educativo, desde una concepción pedagógica, que en condiciones actuales aporte métodos y vías que articule los agentes educativos y los contextos.

La sistematización teórica sobre el proceso de extensión universitaria identificó las funciones de:

-Dinamización, por la relación e interacción entre la universidad y la sociedad, a partir de lo que aportan los contenidos extensionistas desde los procesos académico, investigativo y laboral.

-Gestión, a partir de una articulación coherente, integrada y dialéctica desde los otros procesos, cuyos contenidos se enriquecen, sientan las bases para el desempeño de la formación integral del estudiante y la transformación social.

-Integración, al aglutinar las acciones y operaciones que se ejecutan por los agentes educativos en los diferentes contextos de manera coordinada y participativa, que favorece la labor educativa hacia el estudiante para alcanzar su formación integral.

-Comunicación, al denotar de manera general el impacto y pertinencia de la universidad en la transformación de los contextos y los sujetos a partir de la introducción de los resultados científicos.

$\mathrm{Al}$ atender las funciones declaradas se asume a Alarcón, R. (2015:3) al plantear "las universidades tienen importantes desafíos, incremento del acceso e índices de titulación; preparación en ámbitos científicos, pedagógicos y humanistas; de innovación, de crear, desarrollar la producción científica, lograr la excelencia solo es posible desde una gestión eficiente de su sistema de procesos, donde la extensión universitaria está llamada a ser una pieza clave". Se connota la gestión de la extensión universitaria en la formación integral del estudiante y para evaluar el impacto de la universidad en la transformación de los sujetos y los contextos.

1.2 - Fundamentos pedagógicos del proceso de gestión de la extensión universitaria

En la Declaración Mundial sobre la Educación Superior en el siglo XXI en 1998 se plantea que los centros de educación superior deberán adoptar prácticas de gestión con una perspectiva de futuro que responda a las necesidades de sus entornos. En la teoría, la gestión es la acción y efecto de gestionar, administrar, dirigir, sinónimo de realización, delegación, tramitar, diligenciar, intentar, para conseguir o resolver algo por un colectivo para el logro de los objetivos propuestos. La gestión es un proceso, la administración es una parte de ella relacionada con el manejo y uso de los recursos. Se sustenta en conceptos, teorías, principios, tecnologías, sistemas, estructuras, que dinamizan las instituciones educativas.

Según Ezpeleta, J. (s/a) la gestión educativa constituye un enclave del proceso de transformación, articulador entre las metas y lineamientos del sistema y las concreciones de la actividad escolar, puente hacia las gestiones políticas, administrativas y técnicas. Es un proceso sistemático para el fortalecimiento de las instituciones educativas y sus proyectos, enriquecer sus procesos pedagógicos, directivos, comunitarios, administrativos; conservar la autonomía institucional y responder a las necesidades educativas locales, regionales y mundiales.

Botero, A. (2009:2) sustenta que "La gestión educativa es el estudio de la organización del trabajo en el campo de la educación, influenciada por teorías de la administración, filosofía, ciencias sociales, psicología, sociología y antropología. Se concibe como el conjunto de procesos, toma de decisiones y realización de acciones que llevan a cabo las prácticas pedagógicas, su ejecución y evaluación”. Ideas que se sistematizan en esta investigación desde el proceso de extensión universitaria.

Según Horrutinier, P. (2007:20) "Gestionar el proceso de formación significa organizarlo, planificarlo, desarrollarlo y controlarlo; para asegurar el cumplimiento de los objetivos propuestos. Y esa gestión no es administrativa." Este criterio significa la gestión de la extensión universitaria como un proceso formativo dirigido al cumplimiento de los objetivos del Modelo del Profesional y a la formación integral del estudiante. Desde el colectivo de año académico la gestión es concebida como una relación dialéctica donde se toman decisiones y se ejecutan acciones que conducen a los actores a transformar a los sujetos y contextos, intervienen directivos, profesores, los estudiantes y su familia.

En la gestión de la extensión universitaria se asume como fundamento sociológico la relación socialización e individualización, se asume que la gestión articulada, coherente, integrada y dialéctica, favorece la formación de valores, actitudes, las 
dimensiones del desarrollo: motor, afectivo, cognitivo, comunicativo y social.

Según Batista, T. (2005:12) "el colectivo de año es el órgano básico del proceso de gestión pedagógica en la universidad, la actividad que desarrolla se sustenta en las relaciones entre los componentes del proceso docente educativo, profesores y estudiantes, quienes gestionan didácticamente los contenidos del currículo desde el Enfoque Integral en el proyecto de vida del estudiante, desarrollando la labor educativa desde la instrucción con un enfoque integrador y participativo para lograr el cumplimiento de los objetivos de la formación integral (en las esferas profesional y personal), consolidando la preparación didáctica y pedagógica de sus integrantes para asumir eficientemente sus funciones".

No obstante, es insuficiente la gestión de la extensión universitaria dirigida a cumplir los objetivos formativos, la ejecución y evaluación protagónica de la actividad pedagógica conjunta de profesores, estudiantes, agentes y agencias educativas.

El colectivo de año académico al organizar sus acciones en la estrategia educativa y proyectos extensionistas, debe tener en cuenta los objetivos, contenidos, formas de organización y los contextos. En la literatura (MES, 2014:45) aparece que en extensión universitaria: "sus escenarios educativos por excelencia son la residencia estudiantil, el trabajo comunitario, el área deportiva, el teatro", sin embargo, resulta contradictorio que en la composición del colectivo de año académico, no se incluye el profesor educativo de la residencia estudiantil y el instructor de arte, que desempeñan un papel esencial en la gestión de actividades a partir de las funciones declaradas del proceso de extensión universitaria.

González, M. (2002) plantea que la gestión de extensión universitaria atiende las dimensiones tecnológica y administrativa, donde manifiesta la dialéctica de lo general, lo particular y lo específico, dinamizadas con la promoción sociocultural, relación dialéctica que dota la gestión de peculiaridades propias, sin que pierda su lugar y su esencia, pero circunscrita al desarrollo cultural, sin tener en cuenta lo pedagógico, ni se aportan vías que articulen los otros procesos educativos.

Según Batista, T. (2005:3) el proceso de gestión pedagógica, "garantiza la coordinación, orientación, regulación y evaluación de las acciones didácticas y de carácter socio-psico-pedagógicas que realiza el colectivo de año, mediante el trabajo educativo personalizado y metodológico, relación que determina el carácter consciente, sistémico e integrador de este proceso en la formación integral del estudiante en las esferas profesional y personal del proyecto de vida universitaria, con la optimización de los objetivos en el encargo social, utilización racional de los recursos y una estrategia educativa para mejorar el proceso docente educativo".
El colectivo de año académico organiza la labor educativa sobre la base de los objetivos del año, sin embargo, es insuficiente la coordinación entre los procesos académico, investigativo, laboral, la articulación con los contextos y agentes educativos.

Como síntesis de la categoría gestión y para la comprensión de su nueva visión, se significan las concepciones de los autores consultados:

-Proceso de tipo dialéctico, dialógico, regido por leyes que le permita al estudiante universitario transitar hacia un desarrollo superior y su formación integral.

-Conjunto de procesos, multidimensional y multilateral, aprovecha las influencias de las agencias educativas para realizar su función educativa en relación con los procesos de formación académico, investigativo y laboral.

-Administrar, dirigir, manejo y uso de los recursos, desde una visión pedagógica cuya esencia es formativa.

-Organizarlo, planificarlo, desarrollarlo, controlarlo y evaluarlo; para asegurar el cumplimiento de los objetivos formativos, incorpora procederes para implementarlos en los eslabones de base, con énfasis en el colectivo de año académico.

-Tiene en su esencia las categorías de formación integral, problemas profesionales y contextos de formación que se articulan mediante el método pedagógico.

A partir del análisis de las características de la gestión y su nueva visión, se define el proceso de gestión pedagógica de la extensión universitaria desde el colectivo de año académico como "un proceso orientador, dialógico, multidimensional, dirigido a la formación cultural académica, científica y laboral profesional, cuya dinámica tiene carácter integradora y participativa, favorece la toma de decisiones, realiza acciones para llevar a cabo las prácticas pedagógicas y su evaluación, lo cual permite la formación integral del estudiante, la transformación de los sujetos y los contextos". Batista de los Ríos, D. (2016).

En la gestión pedagógica de la extensión universitaria desde el colectivo de año académico, debe tenerse en cuenta las agencias educativas, fundamentalmente la comunidad vista como contexto donde se genera un proceso de socialización de información y aprehensión de conocimientos, regula el comportamiento social y asegura la formación integral de los mismos. La categoría contexto, del latín contextus, significa lo que rodea a un acontecimiento o hecho. Es un ambiente, un entorno físico, conjunto de fenómenos, situaciones y circunstancias como el tiempo y el lugar no comparables a otras, que rodean o condicionan un hecho, por ejemplo contexto cultural, social, educativo, histórico, económico, psicológico.

Según Centeno, M. A. (2008:1) el contexto 
educativo es "el entorno físico, cognitivo o afectivo, en que se lleva a cabo una acción educativa", aspecto a tener en cuenta desde el colectivo de año académico en la gestión de la extensión universitaria.

Álvarez de Zayas, C. A. (1996a:47), señala "el estudiante aprende, en tanto resuelve un problema, en tanto se estimula, porque como resultado de su labor es más inteligente, en el contexto social está más cerca de los valores que la sociedad establece como modelo". Todo contexto físico o social en que se desarrolla el individuo, es objeto y estímulo de aprendizaje, independientemente que la sociedad no le atribuya de forma implícita o explícita la función de enseñar. Estos espacios físicos donde el estudiante se forma en su vida universitaria se convierten en escenarios educativos; entre ellos están: el aula, el laboratorio, taller, escuelas, empresas, instituciones culturales, comunidades, entre otros, en los cuales adquiere una cultura específica.

En este proceso la cultura juega un papel esencial, seleccionada y sistematizada con sentido pedagógico para incorporar a los planes de estudios, como actividad del hombre al salirse de los límites prefijados por la naturaleza biológica, se distingue porque es una relación sujeto-objeto y sujeto-sujeto.

La educación superior debe priorizar el perfeccionamiento de sus procesos: formación (preservadora de cultura), investigación (creadora de cultura) y extensión (promotora de cultura) con el objetivo de cumplir su misión al favorecer una actitud de cambio y transformación social a través de los profesionales que egresan de las universidades, así como en la interacción dialógica con la comunidad. Es reconocida la cultura como contenido a asimilar, sin embargo, no se aportan vías para articular estos procesos y lograr la transformación de los sujetos y los contextos desde el colectivo de año académico, el cual se centra en relacionar actividades cuyo contenido responde a las diferentes dimensiones de la estrategia educativa de forma aislada $y$ no de manera interrelacionada como un todo.

Según Gainza, M. (2011:4) "La cultura es el cúmulo de conocimientos, capacidades, hábitos y técnicas adquiridos o heredados socialmente, entendida como cultura material. En la misión de las universidades se revela como proceso de formación, resultado del proceso de apropiación, asimilación, elaboración personal, transformación e interiorizada en los sujetos, que integra lo material y espiritual, desde sus procesos sustantivos en particular la extensión universitaria".

El estudiante a partir del conocimiento adquirido es capaz de enriquecerlo y transformarlo, lo que le permite responder a las exigencias sociales, de ahí la relación que se establece entre cultura, educación y sociedad. Esta relación tiene un carácter de proceso, sistémico, progresivo, ascendente, que favorece la transmisión, asimilación, adquisición y apropiación de nuevos conocimientos, habilidades, valores, como síntesis y expresión de la formación integral.

Álvarez de Zayas, C. A. (1995:38) plantea: "el contenido incluye el sistema de conocimientos como reflejo del objeto de estudio y el sistema de habilidades que encierra el conjunto de relaciones del hombre con ese objeto y de los hombres entre sí: relaciones sociales, expresa la riqueza, diversidad y la naturaleza multidimensional del proceso, el carácter académico refleja contenidos abstractos, básicos fundamentales".

Criterio que sistematiza Álvarez de Zayas, R. M. (1997:43) al plantear: "en el contenido se sintetiza una parte de la cultura que se selecciona con criterios pedagógicos con el propósito de formar integralmente al educando". El contenido posee un carácter activo, capaz de transformarse, relacionado con la esencia de la cultura, lo que favorece la formación integral del estudiante.

Cueba, J. L., Ávila, Y. C., Valledor, R. (2016:20) refieren que "la selección, interpretación, procesamiento, elaboración, comunicación y valoración de los contenidos, contribuye a la formación integral". Es vital aprovechar las potencialidades de los contenidos que se aportan. Desde lo sociológico, la gestión pedagógica de la extensión universitaria desde el colectivo de año académico debe atender el proceso formativo en su integralidad, al presentarse de manera multidimensional favorece la formación integral del estudiante por lo que requiere actualmente una nueva modelación.

En la Conferencia Regional sobre Políticas y Estrategias para la Transformación de la Educación Superior en América Latina y el Caribe (s/a), se declaró que las tareas de extensión y difusión deben ser parte importante del quehacer académico, la integración del proceso académico y extensionista influyen en la formación de una cultura académica como elemento de la formación integral. Se impuso la actualización de las prácticas pedagógicas, al atender el carácter integral de la gestión de la extensión universitaria.

Tunmermann, C. (2003), enfatiza en el carácter comunicacional de la extensión universitaria que debe existir hacia la comunidad universitaria y a la sociedad, ya que esta proporciona elementos técnicos, científicos y artísticos, necesarios para la realización personal y colectiva, estructurados con equipos interdisciplinarios.

La universidad, al divulgar sus resultados en respuesta a las exigencias sociales, favorece la transformación de los contextos y fortalece la relación universidad-sociedad. Sin embargo, aunque se han dado pasos al incorporar a los estudiantes a los proyectos comunitarios, tareas de impacto, este requiere nuevas vías en el que se incluya el uso de las 
tecnologías, propuestas que fortalecen el proceso investigativo, que según Addine, F. (2006:7) "es el proceso que deviene como línea directriz de toda la actividad humana, por su esencia constituye una fuente inagotable de conocimientos, de desarrollo de habilidades para la solución de problemas y obstáculos que surgen en la vida profesional.

Desde un enfoque investigativo se forma la conciencia crítica, el pensamiento flexible, alternativo; que potencia en el estudiante universitario recursos que lo ayudan en la solución de problemas profesionales". Desde lo sociológico se forma una cultura científica, el estudiante es capaz de transformar el contexto donde interactúa y muestra una formación integral.

En los contextos, para la asimilación de la cultura el estudiante interactúa con los problemas profesionales, el problema como categoría pedagógica según Matos, E., Cruz. L. (2011:49) debe ser "integrador entre lo fáctico y lo teórico, abierto a una diversidad de posibles alternativas de investigación", lo cual presupone la transformación del contexto diverso y complejo educativo en que se actúa.

Según Trujillo, Y. (2014:61), “es una contradicción que genera la necesidad de actuación del futuro profesional, contribuyen a un proceso de conocimiento más dinámico, reflexivo, crítico, problematizado,motivacional-

profesional,autorreflexivo, favorecen el desarrollo y conducen al logro de las aspiraciones sociales." Los estudiantes se enfrentan a diversos problemas profesionales que reflejan la realidad social, su connotación está en dependencia de los contextos en los que interactúa, donde confluyen los agentes, agencias educativas y escenarios educativos.

El estudiante debe aportar soluciones científicas en las que integre los contenidos recibidos desde lo académico, investigativo, laboral, los métodos profesionales en su futura actividad profesional. Estos se manifiestan en el modo de desempeñarse, que pueda crear y transformar, lo cual favorece su formación integral. La gestión pedagógica de la extensión universitaria implica un mayor reto al colectivo de año académico, a partir de estas condiciones y las particularidades de la carrera, debe potenciar vías de solución desde los procesos universitarios con un carácter pedagógico, en vínculo con el contexto como fuente de cultura.

Según Sanz, N., López, J. A. (2012:41) "la cultura científica es una variedad de la educación en valores, que facilita que los educandos valoren y participen en el contexto, de las dinámicas propias de la sociedad del conocimiento, capaces de desarrollar opiniones personales críticas a partir de la habilidad de poder valorar la información a la que tienen acceso. No se restringe a un conjunto de saberes científicos y destrezas tecnológicas, su significatividad está vinculada a su potencial para generar decisiones y acciones ciudadanas motivadas por consideraciones sociales y humanísticas".

La gestión pedagógica de la extensión universitaria, desde el colectivo de año académico con sus agentes y agencias de socialización, deben formar en el estudiante una cultura que permita la reflexión, revisión y proyección crítica de los vínculos entre teoría y práctica, a partir de lo vivencial, lo empírico y lo investigado, tener en cuenta los intereses y necesidades para consolidar la socializaciónindividualización, materializada en su desempeño laboral.

Lo laboral es un proceso poco sistematizado, aspecto contradictorio, si se trata de formar hombres que se transformen como seres sociales y transformen a la sociedad mediante el trabajo. Según Álvarez de Zayas, C. A. (1995) el componente laboral integra todos los contenidos y lo acerca a la futura actividad profesional, su contenido es el propio de la actividad productiva y los servicios.

En la gestión pedagógica de la extensión universitaria desde el colectivo de año académico, se sistematizan estos contenidos para contribuir a la formación de una cultura laboral, definida como conjunto de principios y valores con los que nos conducimos, el desempeño en el trabajo, cumplimiento de reglas en los contextos de actuación profesional, con principios y valores que definen nuestra actitud y conducta.

En el colectivo de año académico son propuestas acciones para el desarrollo del proceso laboral, pero solo desde las actividades de la práctica pre profesional según el objeto de estudio de la profesión, lo cual es en cierta medida reduccionista, ya que el estudiante se forma en la actividad psíquica y física, por tanto no debe circunscribirse solo a lo laboral. Espinosa, J. A. (2008:62) expresa: "la cultura laboral en el contexto sociocultural laboral es la expresión de la vinculación y aplicación de lo adquirido o construido en la universidad desde el punto de vista académico e investigativo, es la propia formación académica".

La gestión pedagógica de la extensión universitaria desde el colectivo de año académico es el proceso donde ocurre la transmisión, aprehensión y difusión de una cultura laboral formativa, contiene las creaciones del hombre, la dinámica de la actividad con los objetos, los sujetos, debe apuntar a la transformación que ocurre en el estudiante en los ámbitos de formación para que alcance los objetivos profesionales y su formación integral.

La cultura laboral debe atender no solo a la actividad laboral propia, sino a una cultura profesional que constituye modo de actuar y pensar específicos, de acuerdo a condiciones determinadas. Pérez, A. (2009:41) expresa que la cultura laboral es "un proceso de producción de significados en el contexto de relaciones humanas, integración de contenidos, 
solución a problemas que se presentan en la vida cotidiana y la transformación del entorno social de manera flexible y creadora, a partir de asumir los cambios en el universo laboral desde una perspectiva humanista, con la preparación en, desde y para el trabajo."

En la gestión pedagógica de la extensión universitaria desde el colectivo de año académico se debe tener en cuenta este aspecto poco sistematizado, al tener la cultura laboral un carácter continuo en la creación de valores materiales y espirituales, un modo innovador de producirlos, aplicarlos a la vida y afirmarlos en el contexto socio laboral para transformar la sociedad.

La gestión pedagógica de la extensión universitaria desde el colectivo de año académico debe atenderse desde una concepción científica, transformadora sin jerarquizar lo administrativo. Todo el sistema de influencias debe ser puesto en función de la formación integral del estudiante, donde los sujetos en sus diferentes interacciones, reconstruyen y se desarrollan culturalmente, es corroborada la influencia de lo social en lo individual, fundamento psicológico de la teoría histórica cultural de Vygotsky, L. S. (1987), al concebir al hombre como un producto del desarrollo histórico social mediatizado culturalmente, desde esta posición el contexto escolar (comunidad universitaria), familiar (familia del estudiante) y comunitario (sociedad), actúa como fuente de desarrollo educativo y profesional.

Es esencial el diagnóstico para conocer el desarrollo actual y ampliar los límites de la zona de desarrollo próximo o potencial, a partir de actividades extensionistas que tengan en cuenta los objetivos del Modelo del Profesional, los problemas profesionales, potencialidades de los contextos, las agencias y agentes educativos, para lograr un modo de actuación en correspondencia con el objeto de la profesión y los objetivos educativos.

En la interiorización se pone de manifiesto la ley genética general del desarrollo psíquico de Vigostki, L. S. (1987), sobre el papel de lo social y su influencia en lo individual en el proceso de desarrollo del estudiante, en el tránsito de lo social interindividual o inter-psicológico y posterior en el plano intrapsicológico cuyo desarrollo es alcanzado, en tanto adquiera la formación integral. Desde lo psicológico, la gestión pedagógica de la extensión universitaria está condicionada por las necesidades sociales de los estudiantes, que pueden ser académicas, profesionales, científicas, laborales, artísticas.

Esta gestión pedagógica mediada por las relaciones sociales y la cultura como contenido que emana de cada uno de los procesos de la formación académica, laboral e investigativa, favorece la incorporación en el plano individual del sistema de conocimientos, habilidades profesionales, investigativas, laborales, valores y modo de actuación, por lo que en la relación sujeto (profesor)- medio (gestión)-objeto (cultura) y sujeto (estudiante)-medio (gestión)-sujeto (agentes educativos de la comunidad universitaria) se manifiestan condiciones para la formación integral, mediante la realización de actividades que atiendan las necesidades, intereses y motivaciones de los estudiantes concebidas desde el colectivo de año académico.

En la mediación se concibe la relación sujetoobjeto como interacción dialéctica, producida por una mutua transformación mediada por los instrumentos socioculturales y la comunicación en un contexto histórico determinado. Vigotsky, L. S. (1987), destaca el sistema de configuraciones psicológicas de distintos grados de complejidad como el nivel regulador superior de la actividad, mediada en procesos conceptuales, valorativos, procedimentales e interdisciplinarios realizados.

Esta teoría sustenta la gestión pedagógica de la extensión universitaria desde el colectivo de año académico, que pone en el centro al estudiante como sujeto que asimila la cultura, se transforma mediante las vías y formas que ejecute para acceder a la misma durante su actividad, designa el hombre socialmente histórico determinado y portador de la práctica social; favorece la motivación, la innovación, creación, investigación y la comunicación; que pasa al plano individual lo que hace que se transforme, desarrolle y alcance una formación integral.

En la gestión pedagógica de la extensión universitaria desde el colectivo de año académico, es evidente la relación gestión-actividad-formación integral, donde se articula lo académico mediante lo cognitivo, como forma esencial de actividad espiritual del hombre, condicionada por la práctica, que refleja principios, leyes, categorías, teorías, estas se ponen de manifiesto en la actividad en tanto reflejan el objeto, el conocimiento dado por las contradicciones, el desarrollo originado a partir del desconocimiento que tiene el estudiante para alcanzar su formación integral, capaz de satisfacer las exigencias sociales desde su desarrollo individual, adaptándose a los avances de la ciencia y la tecnología.

Mediante la actividad práctica se enfrenta a la necesidad social, desde el plano académico, laboral, investigativo, establece los mecanismos de solución, es transformado el estudiante y la comunidad, vista como contexto de formación para realizar la labor educativa, que evidencia la relación universidad - sociedad. La actividad según Valledor, R. y Ceballo, M. (2006:6), "es una categoría filosófica que se caracteriza por la relación dinámica del hombre con el mundo; mediante la actividad el hombre conoce y transforma la naturaleza".

Esta favorece la interacción entre los problemas profesionales que el currículo propone como campo de investigación y los que enfrenta en su actividad profesional cotidiana, propicia el vínculo entre lo 
colectivo e individual al atender situaciones concretas de desarrollo.

La actividad valorativa es el eslabón entre el conocimiento y la práctica, contradicción entre lo que conoce y debe hacer, valoración dirigida a lo que es significativo socialmente positivo para él, influye en las necesidades, preferencias, pero a la vez es objetivo, se aprende al establecer relaciones significativas. La apropiación de los significados está mediatizada por necesidades y motivos que le confieren al contenido un sentido personal. Se aprende al establecer relaciones significativas, para que sea duradero el aprendizaje, ha de ser significativo.

Por aprendizaje significativo es asumido aquel que parte de conocimientos, actitudes, motivaciones, intereses y experiencia previa del sujeto, lo que hace que el nuevo contenido (cultura) tenga sentido. Lo significativo acontece al vincular los nuevos conocimientos con la cultura previamente establecida, la experiencia práctica, lo afectivo motivacional, aspectos que en su conjunto favorecen la formación integral del estudiante.

Proceso que asume la universidad como gestora del conocimiento, a partir de las potencialidades de los contextos, el papel de agentes y agencias educativas, pero es necesario para la gestión pedagógica de la extensión universitaria desde el colectivo de año académico, un método que articule los elementos mencionados con los procesos académico, investigativo y laboral, que según Valledor, R, y Ceballos, M. (2006:12): "es una forma organizada y eficiente de obtención de conocimientos, y adquisición de cultura", que favorecerá la formación integral del estudiante.

Los métodos como categoría pedagógica dirigidos hacia la educación son las vías que se siguen para lograr el objetivo educativo planteado por la sociedad, las tareas específicas a desarrollar en condiciones desarrolladas del proceso educativo. Es un sistema de actividades sucesivas que conducen a un resultado consecuentemente con los objetivos planteados, la aplicación del método implica una serie de operaciones que compone el procedimiento que puede ser adecuado o no a la situación educativa de referencia.

Es pertinente una sistematización teórica que proponga un nuevo proceder desde el colectivo de año académico, para lograr una gestión pedagógica eficiente y la formación integral del estudiante. Desde la investigación se debe formar un profesional creativo, crítico, innovador, vinculado a la ciencia como actividad enfocada hacia la producción, difusión $\mathrm{y}$ aplicación del conocimiento, llevar a cabo la investigación científica, sustentada en un método científico, que según Valledor, R. y Ceballo, M. (2006:17), "no puede operar en un vacío de conocimiento, requiere de un sistema de conocimientos previos que pueda reajustarse".
Álvarez de Zayas, C. A. (1995a:4) enfatiza "el método garantiza en su dinámica, la apropiación del contenido, el logro del objetivo. Es flexible, se adecua a las condiciones para alcanzar el objetivo. El trabajo es método, el más significativo para alcanzar la formación". La preparación del estudiante debe enfocarse hacia los problemas profesionales y las exigencias sociales, la respuesta a estos será la expresión de su formación integral.

Martí, J. (1978:53) expresó: "Puesto que a vivir viene el hombre, la educación ha de prepararlo para la vida. En la escuela se ha de aprender el manejo de las fuerzas con que en la vida se ha de luchar". Desde esta concepción pedagógica la gestión de extensión universitaria debe ser reconocida como un proceso formativo, educativo y social, que no acontece aisladamente sino dentro de un espacio social, extendida a diferentes contextos de formación que dinamiza el vínculo universidad - sociedad. El estudiante para alcanzar una formación integral debe integrar contenidos académicos y extensionistas, que al aplicarlos en la práctica le permita resolver problemas profesionales, aspecto que aún es necesario sistematizar.

El permitir que el sistema de influencias educativas actúe espontáneamente, es negar la capacidad integradora y proyectiva de la universidad ante el reto de preparar al hombre para la vida social, objetivos aprendidos en la interacción en la escuela, con la familia, la comunidad, ya que los conocimientos se aprenden en la sociedad para verterlos luego en la misma. La extensión universitaria está en constante transformación, revela problemas conceptuales y prácticos que exigen un redimensionamiento en sus concepciones y métodos que la proyecten hacia la sociedad, y que a la vez garanticen la pertinencia de este proceso formativo desde una concepción pedagógica para transformar sujetos y contextos.

Álvarez de Zayas, C. A. (1995:58) sustenta: "la formación es un proceso totalizador que integra la educación, la instrucción y el desarrollo, en procesos escolares y no escolares, cuya función es la preparación del hombre como ser social", criterio que reafirma a la gestión pedagógica de la extensión universitaria como proceso formativo, al potenciar el desarrollo de la universidad en la sociedad.

La universidad es la encargada de llevar el acervo cultural que el hombre en su actividad ha logrado a lo largo de la historia, y desde el punto de vista académico se manifiesta en la relación establecida entre profesor (enseñanza)-cultura (contenido) y estudiante (aprendizaje), en la formación de las nuevas generaciones, de ahí la relación cultura y formación.

Debe ser promotora de nuevos conocimientos, nuevas formas de transformar la realidad y su entorno, según Horrutinier, P. (2007:7) "Sin investigación científica no es posible hablar de la verdadera 
formación de un profesional en ninguna carrera universitaria, del mismo modo que la labor investigativa, en las universidades, se soporta en buena medida en los procesos de formación de pregrado y posgrado." Criterio que revela la relación de investigación, cultura y formación, aspecto esencial en la gestión pedagógica de la extensión universitaria.

La formación y creación de la cultura sostienen su relación con la promoción y divulgación, surge así la extensión universitaria como concepto y eslabón en la tríada preservación-desarrollo-promoción. Las universidades son promotoras de la cultura en su sentido más amplio, utilizan como método la promoción para llevar toda esa cultura a la sociedad a través de cada uno de sus procesos.

Según Addine, F. (2006:7) "La integración de lo académico, lo laboral y lo investigativo contribuye al desarrollo de la autodeterminación y aporta tanto el aspecto intelectual como el aspecto afectivo volitivo". Alarcón, R. (2015:11) refiere al respecto que "la integración de las funciones universitarias, formación, investigación, extensión, logra la pertinencia en función del desarrollo y la formación integral del estudiante".

Según Álvarez de Zayas, C. A. (1995:40) "la formación tiene un carácter inmediato, vinculado con el desarrollo del pensamiento: la instrucción, y otro más trascendente relacionado con la conformación de valores, convicciones, sentimientos: la educación". Ambos aportan resultados diferentes que se complementan en un mismo proceso, el punto de partida de la formación es la medida de la significación que el contenido tenga para el estudiante. Betto, F. (2014:14) expresó "el proceso de formación no se limita a trasmitir conocimientos, debe buscar el hombre nuevo".

Álvarez de Zayas, R. M. (1997:44) fundamenta que "la formación integral de la personalidad del educando en sus dimensiones cognoscitivas y valorativas; se constituyen a partir de los contenidos de tipo cognoscitivos, procedimentales (intelectuales y motores) y actitudinales. Los objetivos de la educación no son suma de modalidades educativas, sino elementos inseparables de una integración armónica del carácter del educando". Horrutinier, P. (2007:3) plantea "es el quehacer de las universidades en la formación de valores en los profesionales de forma más plena, dotarlos de cualidades de alto significado humano, capaces de comprender la necesidad de poner sus conocimientos al servicio de la sociedad, en lugar de utilizarlos para beneficio personal".

Estas definiciones de formación integral permiten identificar sus rasgos o cualidades, ellas son:

-Tiene carácter de proceso. Se basa en conocimientos científicos (conceptos, teorías, enfoques, paradigmas), incluye actitudes, normas, valores, productos de la acción humana, modo (modelos, métodos) de pensamiento y actuación aceptado por la sociedad.

-Integra de forma armónica los objetivos de la educación en el sujeto con sus particularidades individuales en interrelación con la sociedad. Facilita pedagógicamente el acceso al patrimonio cultural de la nación y de la humanidad. Estos criterios son contextualizados a partir de las condiciones históricas concretas de la nueva universidad en el Documento para el diseño de los planes de estudio "E" (MES, 2016:9), que plantea que la formación integral se refleja en "graduados con un sólido desarrollo político desde los fundamentos de la ideología de la Revolución cubana; dotados de un amplia cultura científica, ética, jurídica, humanista, económica y medio ambiental; comprometidos y preparados para defender la Patria con argumentos propios, competentes en el desempeño profesional y el ejercicio de una ciudadanía virtuosa".

Estos elementos concatenados entre sí constituyen un sistema complejo, cuyo resultado es su capacidad de contribuir de forma creadora a encontrar solución a los problemas de la práctica. La gestión pedagógica de la extensión universitaria desde el colectivo de año académico es fundamental en el cumplimiento de estos objetivos, la transformación de los sujetos, contextos, y en la formación integral del estudiante.

\section{Conclusiones}

La sistematización teórica reveló que el proceso de gestión de la extensión universitaria requiere una concepción pedagógica. Esta labor le corresponde a los profesores y no a una estructura específica, aún existe un predominio de la orientación hacia lo artístico deportivo, en detrimento de la investigación, lo académico y laboral, aspecto que incide en su fragmentación, en particular en los contenidos, vías y formas de la gestión.

Este proceso está en constante transformación, al revelar problemas conceptuales y prácticos que exigen un redimensionamiento en sus concepciones y métodos que la proyecten hacia la sociedad, es necesario avanzar en la búsqueda de conocimientos que garanticen pertinencia del proceso formativo universitario y nuevas vías para su gestión con calidad, desde una concepción pedagógica, como proceso formativo, que ocurre no de forma aislada, sino dentro de un espacio social que se extiende a diferentes contextos de formación y favorece la formación integral del futuro profesional. 


\section{Referencia bibliográfica}

ADDINE, F. Modo de actuación profesional pedagógica. De la teoría a la práctica. Editorial Academia. La Habana. 2006.

ALARCÓN, R. Conferencia inaugural en el XIII Congreso Latinoamericano de Extensión Universitaria. La Habana. 2015.

ÁLVAREZ DE ZAYAS, C. A. La escuela en la vida. Editorial Félix Varela. La Habana. 1995.

ÁLVAREZ DE ZAYAS, R. M. Hacia un currículo integral $\mathbf{y}$ contextualizado. Editorial Academia. La Habana. Cuba.1997.

BATISTA, T. Propuesta de gestión pedagógica del año académico. Metodología de instrumentación en la carrera de Agronomía en la Isla de la Juventud. [tesis doctoral]. Isla de la Juventud. Cuba. 2005.

BATISTA, D. Gestión de la extensión universitaria por el método participativo integrador extensionista y sus procedimientos. Revista Dilemas contemporáneos: Educación, Política y Valores. Año IV. Publicación No.2. México. 2016.

BETTO, F. El papel del educador en la formación política de los educandos. Editorial Félix Varela. Cuba. 2014.

BOTERO, C. A. Cinco tendencias de la gestión educativa. Revista Iberoamericana de Educación. No. 49/2. Colombia. 2009.

CENTENO, A. M. La importancia del contexto en la enseñanza de la medicina. Un concepto elusivo y muchas veces olvidado. Revista Argentina de Educación Médica. Vol. 2. No. 2. Argentina. 2008.

\section{CONSTITUCIÓN DE LA REPÚBLICA DE CUBA. Cuba.} 1992.

CONGRESO UNIVERSIDAD 2014. Relatoría del XII Taller Internacional de Extensión Universitaria. La Habana. Cuba. 2014.

CUEVA, J. L., ÁVILA, Y. C. El uso de las tecnologías, de la información y las comunicaciones en la gestión del conocimiento: una necesidad en la escuela primaria. Revista Opuntia Brava. Vol.13. No.4. Las Tunas. Cuba. 2015.

DEL VALLE BLOHM, N. C. La gestión de la cultura extensionista desde las instituciones de Educación Superior. [tesis doctoral].Santiago de Cuba. Cuba. 2009.

ESPINOSA, J. A. Gestión de la cultura profesional en la educación superior. [tesis doctoral]. Granma. Cuba. 2008.
GAINZA, M., Paz, A. Fundamentos teóricos desde la extensión universitaria en el proceso de formación de los profesionales pedagógicos en el contexto de la universalización. Cuadernos de Educación y Desarrollo. Vol.3 No. 27. España. 2011.

GONZÁLEZ, M., GONZÁLEZ， G. R. ¿Extensión universitaria, proyección social o tercera misión?. Una reflexión necesaria. Revista Congreso Universidad. Vol. II. No. 2. Editorial Universitaria Félix Varela. Cuba. 2013.

GONZÁLEZ, M. Modelo de gestión de la extensión universitaria para la Universidad de Pinar del Río. [tesis doctoral]. Pinar del Rio. Cuba. 2002.

HORRUITINER, P. La universidad cubana: El modelo de formación. Revista Pedagogía Universitaria. Vol. 12. No.4. La Habana. Cuba. 2007.

MARTÍ, J. Obras completas. Tomo 13. Cuba. 1978.

MATOS, E., CRUZ, L. La práctica investigativa, una experiencia en la formación doctoral en Ciencia Pedagógica. Órgano Editor Ediciones Universidad de Oriente. Ministerio de Educación Superior de Cuba. 2011.

MINISTERIO DE EDUCACIÓN SUPERIOR. Perfeccionamiento del sistema de gestión del proceso de formación integral del estudiante universitario en el eslabón de base. (Segunda parte). Editorial Universitaria Félix Varela. La Habana. Cuba. 2014

SANZ, N., LÓPEZ, J. A. Cultura científica para el siglo XXI. Revista Iberoamericana de Educación. No. 58. Colombia. 2012.

PÉREZ, A. La cultura laboral en las condiciones del modelo de Secundaria Básica. [tesis doctoral]. Santiago de Cuba. Cuba. 2009.

TRUJILLO, Y. Metodología para el empleo de los medios de enseñanza-aprendizaje en la Disciplina Procesos Constructivos: su contribución a la profesionalización del docente en formación inicial. [tesis doctoral]. Las Tunas. Cuba. 2014.

TUNNERMANN, C. La Universidad latinoamericana ente los retos del siglo XXI. Unión de Universidades de América Latina. Circuito Norponiente SíN. Ciudad Universitaria. México. 2003.

VALLEDOR, R., CEBALLO, M. Metodología de la investigación educacional. Organo Editor Educación Cubana. Ciudad de la Habana. Cuba. 2006.

VIGOSTKI LEV, S. Historia del desarrollo de las funciones psíquicas superiores. Editorial Pueblo y Educación. La Habana. Cuba. 1987. 